\title{
Radio searches for supernovae in nearby starburst galaxies
}

\author{
Megan Argo*, Alan Pedlar, Rob Beswick, Tom Muxlow, Danielle Fenech and Mirza \\ Asif \\ Jodrell Bank Observatory \\ E-mail: mkargo@manchester.ac.uk
}

Over the last four years, we have been using both MERLIN and the VLA to regularly observe a sample of nearby starburst galaxies, looking for new radio supernovae in order to investigate their starformation rates, and monitoring the fluxes of known compact objects within each galaxy. In the course of this project we have observed many supernovae, discovering two, placing upper limits on the early radio emission for several, and producing two of the most detailed radio light curves for type II supernovae. Here we briefly outline some of the recent results, including MERLIN single-baseline observations carried out over September and October 2007.

From Planets to Dark Energy: the Modern Radio Universe

October 1-5 2007

The University of Manchester, UK

* Speaker. 


\section{The Monitoring Programme}

The monitoring programme is designed to investigate the starformation rates in a sample of nearby starburst galaxies. The starformation rate (SFR) is an important parameter which can be measured using a variety of methods. In principle, the supernova rate provides an independent estimate of the SFR, and radio searches for core collapse supernovae have the advantage that extinction is not an issue. The galaxies in the sample were selected from Condon et al [5]: all are starbursts within $30 \mathrm{Mpc}$, have IRAS counterparts and fluxes greater than $90 \mathrm{mJy}$ at $4.85 \mathrm{GHz}$. The sample is observed every three or four months to search for new compact objects. Observing with this frequency easily allows new core collapse events to be spotted as they tend to be radio bright on time-scales of several months to many years. So far, we have completed eleven epochs of observations, detected several RSN events and put limits on the early radio emission for others detected in other wavebands. Table 1 lists the supernovae observed as part of this project over the last four years along with their locations, whether they were detected, and either their peak fluxes or $3 \sigma$ limits in cases where no detection was made.

Most of the observations in the monitoring programme have been carried out using either the VLA or full MERLIN during regular scheduled sessions. The VLA is used when in A or B configurations, MERLIN is used at other times when the VLA is in more compact configurations which lack the required resolution. Some supernovae events have occurred during the MERLIN summer engineering period when few telescopes are available. During these periods we have been using all available antennas to observe these transient events. Often this involves using a single baseline which, while clearly unsuitable for imaging, does allow flux measurements to be made. Such observations have resulted in detections and light curves for several supernovae which otherwise might not have been observed.

\section{Starformation Rates}

A search for new supernovae in a sample of Southern hemisphere galaxies found no events by searching pairs of archival images for sources which appear in the later images[8]. No new sources were detected in the pilot survey, but limits on the supernova rates were calculated for each galaxy in their sample. By using a similar method, estimates for the radio supernova rates in galaxies in the monitoring sample can be determined. First the 'control time' (the time during which a given event could be detected, i.e. the number of years during which the received flux density is above the noise level of the telescope) is calculated. The 'surveillance time' is then the control time multiplied by the optical blue magnitude of the galaxy in units of $10^{10} \mathrm{~L}_{\odot}$. An estimate of the supernova rate is then obtained by dividing the number of supernovae detected per year during the survey by the surveillance time. For the monitoring sample, only two galaxies give results for SN2004dj-like events (a peak of $2 \mathrm{mJy}$ at a distance of $3.3 \mathrm{Mpc}$ ) since most have either zero observed $\mathrm{SNe}$ over the three years of the programme to date, or zero surveillance time due to their distance. For brighter J1048-like events (a $5 \mathrm{GHz}$ estimated peak of $2.4 \mathrm{mJy}$ at $13.3 \mathrm{Mpc}$ ), half the sample have non-zero estimated rates while the rest have upper limits as shown in Table 1. These estimates are similar to previous estimates made using other methods (e.g. $0.05 \mathrm{SNe} /$ year for M82, $[6])$. 


\begin{tabular}{|c|c|c|c|c|c|}
\hline Galaxy & $\begin{array}{c}\mathrm{L}_{\text {blue }} \\
\left(10^{10} \mathrm{~L}_{\odot}\right)(\mathrm{yrs})\end{array}$ & $\begin{array}{c}\text { Control time } \\
(\mathrm{yrs})\end{array}$ & $\begin{array}{c}\text { Surveillance time } \\
\left(\mathrm{RSN} / 100 \mathrm{yrs} / 10^{10} \mathrm{~L}_{\odot}\right)\end{array}$ & $\begin{array}{c}\text { RSN rate } \\
\text { now }\end{array}$ & $\begin{array}{c}\text { RSN rate } \\
\text { after 5 years }\end{array}$ \\
\hline M82 & 0.17 & 50.38 & 8.76 & 3.8 & 2.3 \\
NGC520 & 0.58 & 13.47 & 7.76 & $<4.3$ & $<2.6$ \\
NGC891 & 0.35 & 30.74 & 10.7 & $<3.1$ & $<1.9$ \\
NGC2146 & 0.52 & 21.89 & 11.5 & 2.9 & 1.7 \\
UGC2866 & 0.05 & 8.8 & 0.41 & $<81$ & $<49$ \\
NGC3310 & 0.54 & 23.53 & 12.8 & 2.6 & 1.6 \\
NGC3628 & 0.56 & 30.95 & 17.2 & $<1.9$ & $<1.2$ \\
NGC4038 & 0.56 & 22.83 & 12.8 & 2.6 & 1.6 \\
NGC4631 & 0.91 & 30.95 & 28.0 & $<1.2$ & $<0.7$ \\
NGC6946 & 0.44 & 38.96 & 17.3 & 1.9 & 1.2 \\
\hline
\end{tabular}

Table 1: Galaxies in the monitoring sample together with their calculated supernova rates based on the observations carried out as part of the monitoring programme to date. The RSN rate after five years assumes no further RSN detections in the next year of observations.

\section{Recent Results}

2007gr: type Ib/c SN in NGC1058, discovered optically August 2007. This supernova was bright (magnitude 13.8 at a distance of $9 \mathrm{Mpc}$ ) and has been observed by many groups. The VLA detected early emission at $8.4 \mathrm{GHz}$ [9] while the GMRT found no source at $1.4 \mathrm{GHz}$ [4]. MERLIN observations using the Mk2, Darnhall and Defford telescopes found no source to a $3 \sigma$ limit of $0.43 \mathrm{mJy} / \mathrm{bm}$, to Sept $6^{\text {th }}$ [2]. eVLBI observations detected a $5.6 \sigma$ source at $422 \mu \mathrm{Jy}$ on Sept $7^{\text {th }}$ [7]. MERLIN observations are continuing with all available antennas.

J1120: RSN in NGC3628, discovered by this programme in Dec 2006 [3]. MERLIN observations carried out as part of the monitoring programme showed a new source in this galaxy with a flux density of $1.1 \mathrm{mJy}$ at $5 \mathrm{GHz}$. Further observations in January 2007 failed to detect this source, implying that it decayed rapidly. This source was located in an optically-obscured region of the galaxy and no optical counterpart was seen by other observers.

2006gy: type IIn SN in NGC1260, discovered optically September 2006. Observed with MERLIN as a ToO in May 2007 at 1.6 and $5 \mathrm{GHz}$ but no source was detected to $3 \sigma$ limits of 0.28 and $0.8 \mathrm{mJy} / \mathrm{bm}$ respectively [1].

2006be: type II SN in IC4582, discovered optically March 2006. Observed with MERLIN as a ToO with the full array at $6 \mathrm{GHz}$ in April 2006 but no source was detected to a $3 \sigma$ limit of $0.31 \mathrm{mJy} / \mathrm{bm}$.

\section{Future}

Observations will continue for another year. When completed, in addition to the estimates of starformation rates, this survey will provide information on the long-term variability of known compact sources in the sample galaxies. This project is also a pilot for the upgraded e-MERLIN. Currently, MERLIN observations of transient and variable sources take many hours in order to provide sufficient sensitivity and $u-v$ coverage. When the new system becomes operational, the 


\begin{tabular}{|c|c|c|c|c|}
\hline SN detection & Host galaxy & Distance (Mpc) & Detection? & Flux/limit (at 5GHz) \\
\hline J1038* & NGC3310 & 13.3 & light curve & $2 \mathrm{mJy}$ \\
$2004 \mathrm{am}$ & M82 & 3.2 & $\mathrm{x}$ & $<0.18 \mathrm{mJy} / \mathrm{bm}$ \\
$2004 \mathrm{dj}$ & NGC2403 & 3.3 & light curve & $2 \mathrm{mJy}$ \\
$2004 \mathrm{et}$ & NGC6946 & 5.9 & light curve & $2.5 \mathrm{mJy}$ \\
$2004 \mathrm{gt}$ & NGC4038 & 13.8 & $\mathrm{x}$ & $<0.2 \mathrm{mJy} / \mathrm{bm}$ \\
$2005 \mathrm{~V}$ & NGC2146 & 14.5 & $\mathrm{x}$ & $<0.4 \mathrm{mJy} / \mathrm{bm}$ \\
$2005 \mathrm{cs}$ & M51 & 8 & $\mathrm{x}$ & $<0.2 \mathrm{mJy} / \mathrm{bm}$ \\
$2006 \mathrm{be}$ & IC4582 & 29 & $\mathrm{x}$ & $<0.3 \mathrm{mJy} / \mathrm{bm}$ \\
$2006 \mathrm{gy}$ & NGC1260 & 75 & $\mathrm{x}$ & $<0.18 \mathrm{mJy} / \mathrm{bm}$ \\
$2006 \mathrm{jc}$ & UGC4904 & 23 & $\mathrm{x}$ & $<0.3 \mathrm{mJy} / \mathrm{bm}$ \\
J1120* & NGC3628 & 9 & detection & $1.1 \mathrm{mJy}$ \\
$2007 \mathrm{gr}$ & NGC1058 & 7 & $\mathrm{x}$ & $<0.4 \mathrm{mJy} / \mathrm{bm}$ \\
\hline
\end{tabular}

Table 2: Supernovae observed using MERLIN and the VLA during the monitoring programme so far. Where detections were made, the measured flux density at $5 \mathrm{GHz}$ is given, otherwise the $3 \sigma 5 \mathrm{GHz}$ limit is listed. $\mathrm{SN}$ denoted by * were discovered by this programme.

greater data capacity and wide-band receivers will provide greatly enhanced imaging capabilities. "Snapshot" observations ideal for programmes such as this, which currently take several hours, will be carried out in a matter of minutes.

\section{References}

[1] M. K. Argo, R. J. Beswick, T. W. B. Muxlow, and A. Pedlar, Radio observations of SN 2006gy with MERLIN, Astronomer's Telegram 1084 (2007c), 1.

[2] M. K. Argo, R. J. Beswick, A. Pedlar, and T. W. B. Muxlow, MERLIN observations of SN2007gr, Astronomer's Telegram 1210 (2007a), 1.

[3] M. K. Argo, T. W. B. Muxlow, R. J. Beswick, and A. Pedlar, Possible Radio Supernova in NGC 3628, Central Bureau Electronic Telegram 842 (2007b), 1.

[4] S. Chakraborti, P. Chandra, N. Roy, and A. Ray, Radio monitoring of SN 2007gr by GMRT in the L-band, Astronomer's Telegram 1222 (2007), 1.

[5] J. J. Condon, E. Anderson, and J. J. Broderick, Radio Identifications of Extragalactic IRAS Sources, Astron. J. 109 (1995), 2318-+.

[6] T. W. B. Muxlow, A. Pedlar, P. N. Wilkinson, D. J. Axon, E. M. Sanders, and A. G. de Bruyn, Mon. Not. R. Astr. Soc. 266 (1994), 455.

[7] Z. Paragi, C. Kouveliotou, M. A. Garrett, E. Ramirez-Ruiz, H. J. van Langevelde, A. Szomoru, and M. Argo, e-VLBI detection of SN2007gr, Astronomer's Telegram 1215 (2007), 1.

[8] E. M. Sadler and D. Campbell-Wilson, A search for radio-loud supernovae, Publications of the Astronomical Society of Australia 14 (1997), 159-63.

[9] A. Soderberg, Discovery of Radio Emission from the Type Ibc SN 2007gr, Astronomer's Telegram 1187 (2007), 1. 